\title{
BUSINESS MODELS IN THE CONDITIONS OF INCREASING COMPLEXITY OF THE BUSINESS ENVIRONMENT
}

\author{
Elena V. Shirinkina \\ Surgut State University, Surgut, Russian Federation
}

\begin{abstract}
The relevance of the study is due to the fact that in the increasingly complex business environment, companies today more than ever need a simple and flexible tool to manage innovations, to build effective business models in a timely manner. The purpose of the study is to present updated business models in the context of the digital economy development. The empirical base was the research of $\mathrm{H}$. Mendelssohn, A. Osterwalder, as well as analysts of the best business practices of Alibaba Group, Apple, Uber, AirbnB, Amazon, Facebook, Google. The author presents the transformation of business models and their adaptation to a new, changed environment, as well as business models that are relevant in innovation management. The practical significance of the study lies in the fact that the results obtained will allow companies to achieve business success, getting a greater effect from the application of this business model by combining it with other tools. This will help companies to obtain the optimal set of ideas and associated potential risks at the output in order to identify the most promising business development ideas and prioritize.

Key words: business models, companies, management, innovation, digital economy, tools.

Citation. Shirinkina E.V. Business Models in the Conditions of Increasing Complexity of the Business Environment. Vestnik Volgogradskogo gosudarstvennogo universiteta. Ekonomika [Journal of Volgograd State University. Economics], 2021, vol. 23, no. 1, pp. 121-130. (in Russian). DOI: https://doi.org/10.15688/ek.jvolsu.2021.1.10
\end{abstract}

УДК 336.1

ББК 65.290 .2

Дата поступления статьи: 24.12.2020

Дата принятия статьи: 12.01.2020

\section{БИЗНЕС-МОДЕЛИ В УСЛОВИЯХ ВСЕ ВОЗРАСТАЮЩЕЙ СЛОЖНОСТИ БИЗНЕС-СРЕДЫ}

\author{
Елена Викторовна Ширинкина \\ Сургутский государственный университет, г. Сургут, Российская Федерация
}

\begin{abstract}
Аннотация. Актуальность исследования обусловлена тем, что в условиях все возрастающей сложности бизнес-среды сегодня компаниям как никогда необходим простой и гибкий инструмент, чтобы управлять инновациями, своевременно выстраивать эффективные бизнес-модели. Цель исследования - представлены актуализированные модели бизнеса в контексте развития цифровой экономики. Эмпирической базой послужили исследования Х. Мендельсона, А. Остервальдера, а также аналитика лучших бизнес-практик Alibaba Group, Apple, Uber, AirbnB, Amazon, Facebook, Google. Автором представлена трансформация бизнес-моделей и адаптация их к новой, изменившейся среде, а также бизнес-модели, актуальные в управлении инновациями. Практическая значимость исследования заключается в том, что полученные результаты позволят компаниям добиваться успеха в бизнесе, получая больший эффект от применения данной бизнес-модели комбинируя ее с другими инструментами. Это поможет компаниям получить на выходе оптимальный набор идей и связанных с ними возможных рисков, чтобы определить наиболее многообещающие идеи развития бизнеса и расставить приоритеты.

Ключевые слова: бизнес-модели, компании, управление, инновации, цифровая экономика, инструментарий.

Цитирование. Ширинкина Е. В. Бизнес-модели в условиях все возрастающей сложности бизнес-среды // Вестник Волгоградского государственного университета. Экономика. - 2021. - Т. 23, № 1. - С. 121-130. DOI: https://doi.org/10.15688/ek.jvolsu.2021.1.10
\end{abstract}




\section{Введение}

Новые технологии стремительно меняют бизнес-среду. Сегодня потребитель суперинформирован, суперподключен и обладает возможностью поделиться с огромной аудиторией своими впечатлениями об опыте взаимодействия с любой компанией на рынке. Все это создает для бизнеса сложности, но и открывает возможности.

Прежде чем говорить о новых типах бизнес-моделей, вспомним основные сущности, которые определяют саму концепцию бизнес-модели. Это, во-первых, способ создания ценности (товара или услуги), во-вторых, модель извлечения прибыли, и в-третьих, логика бизнеса.

Сегодня трудно найти бизнес, менеджмент которого не думал бы о трансформации своей бизнес-модели. Это объяснимо, однако, внедряя IT-технологии, важно помнить о своих ключевых компетенциях, о том, как измененная модель позволит лучше их использовать. Google и Facebook поражают воображение тем, что построили бизнес на основе услуг, которых не существовало до появления Интернета; Apple, например, так и осталась производителем компьютеров, гаджетов и программного обеспечения, просто усилила свою модель online-платформой, предназначенной для обслуживания своих клиентов - обладателей девайсов Apple.

Хаим Мендельсон, профессор Стэнфордской бизнес-школы, утверждает, что, рассуждая о будущем, имеет смысл обратить внимание на три архетипа бизнес-моделей, каждый из которых характеризуется своей собственной логикой [Абанина, 2019, с. 24]:

1. Первый основывается на долгосрочных доверительных отношениях с клиентами. В этом случае все решения подгоняются под требования клиентов усилиями фронт-офиса. Пример такой модели - The Ritz-Carlton, управляющий роскошными пятизвездочными отелями и курортами по всему миру.

2. В основе второго лежит операционная эффективность. Она, в свою очередь, базируется на улучшенных процессах бэк-офиса. Типичный представитель использования этой модели - сеть супермаркетов Walmart, пред- лагающая покупателям качественные товары по низким ценам.

3. Третий ориентируется на создание ценности путем координации фронт-офиса и бэкофиса. Именно здесь открываются возможности для создания инновационных бизнесмоделей, а именно цифровых платформ. Суть платформы (например, еВау) в том, что она делегирует непосредственное создание ценностей другим участникам (производителям товаров и услуг), сама же координирует действия продавцов и покупателей, направляет бизнес-процессы и сокращает затраты на транзакции. Ее задача - усовершенствование самой платформы, работа над привлечением клиентов и создание для них дополнительных источников ценности.

Хотя сама концепция бизнес-модели актуальна для любого бизнеса, в последнее время чаще всего о ней вспоминают, когда речь заходит о стартапах в IT-сфере. Это неудивительно, так как именно информационные технологии ускорили процесс развития бизнесмоделей. Создание прототипов и проведение тестирований, которые раньше стоили сотни тысяч долларов и длились месяцами, теперь может быть завершено в течение недели и стоить значительно дешевле.

Хаим Мендельсон предлагает обратить внимание на три разработки, которые следует иметь в виду при поиске новой бизнес-модели: во-первых, слияние виртуальных и физических личностей, во-вторых, сближение моделей и реальности, и в-третьих, конвергенция атомов и байтов. Хаим Мендельсон предсказывает, что три типа бизнес-моделей будут играть важную роль в использовании IT [Мариничев, 2015, с. 75]:

1. Агенты, отвечающие за формирование отношений с клиентами, будут фокусироваться на изучении текущих и прогнозировании будущих предпочтений потребителей и на помощи в процессе выбора альтернативных решений. Они станут цифровыми представителями клиентов на рыночных площадках, используя их данные, будут находить и формировать наилучшие предложения.

2. Координаторы цепочек ценности будут сопоставлять спрос и предложение, моделируя решения, которые уже используются в существующих продуктах, и новые раз- 
работки, основанные на предпочтениях клиентов. Они научатся собирать уникальные кастомизированные решения, будут принимать участие в инновациях, управляемых данными клиентов.

3. Производители и поставщики будут специализироваться на разработке и продаже физических и онлайн-продуктов, которые разрабатываются и тестируются при помощи цифрового моделирования и дорабатываются на основе быстрой реакции потребителей.

Базовые бизнес-модели тоже сохранятся, но потребуют постоянных нововведений с учетом влияния времени и развития новых технологий.

Цель исследования - представить актуализированную модель бизнеса в контексте развития цифровой экономики. Эмпирической базой послужили исследования Х. Мендельсона, А. Остервальдера, а также аналитика лучших бизнес-практик Google, Facebook, Apple. Представленная бизнес-модель позволит ее эффективно использовать в управлении инновациями. Практическая значимость исследования заключается в том, что полученные результаты позволят компаниям добиваться успеха в бизнесе, получая больший эффект от применения данной бизнес-модели, комбинируя ее с другими инструментами. Это поможет компаниям получить на выходе оптимальный набор идей и связанных с ними возможных рисков, чтобы определить наиболее многообещающие идеи развития бизнеса и расставить приоритеты.

\section{Методика}

Современный мир стремительно меняется, бизнес меняется вместе с ним, и сегодня компаниям как никогда необходим простой и гибкий инструмент, чтобы управлять инновациями, своевременно менять и выстраивать бизнес-модель, позволяющую преуспеть в условиях все возрастающей сложности бизнес-среды. В последнее время наиболее популярным стал инструмент, получивший название «Business model Canvas» (далее ВMC) Александра Остервальдера [IВМ ...].

На сегодняшний день имеется множество трактовок того, как его применять, но все они основаны на четырех основных элементах:

- ценностное предложение;

- потребители и взаимодействие с ними;

- бизнес-структура компании, ее внешние и внутренние ресурсы;

- финансовые потоки (рис. 1).

В центре ВМС находится ключевой и, пожалуй, самый сложный для любого бизнеса элемент - ценностное предложение. Чтобы его понять и описать, необходимо ответить на ряд ключевых вопросов: в чем ценность нашего продукта или нашей услуги для потребителя? какие проблемы клиентов мы решаем? что ценного в нашем предложении? что мы можем предложить для каждого сегмента потребителей? Для каждого сегмента потребителей необходимо сформулировать ответы на эти вопросы, что на самом деле очень непросто, особенно если речь о новых продуктах или рынках.

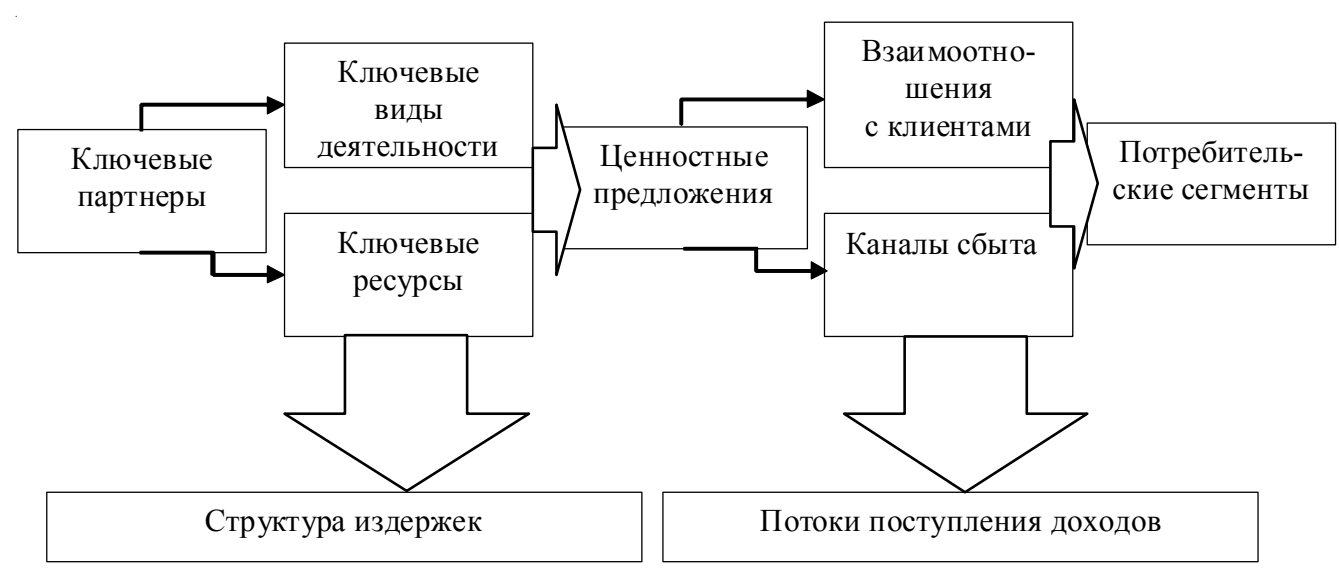

Рис. 1. Шаблон бизнес-модели ВМС

Примечание. Составлено автором на основе источников: [Дунаев, 2020, с. 157; Благов и др., 2020, с. 157]. 
Справа расположен блок, состоящий из трех подразделов, который посвящен потребителям и способам взаимодействия с ними.

1. Потребительские сегменты:

- для кого мы работаем?

- кто для нас самый важный клиент?

2. Взаимоотношения с клиентами:

- каковы наши отношения с клиентами в каждом из сегментов?

- каких взаимоотношений ожидают от нас клиенты? дель ?

- как они интегрированы в бизнес-мо-

- насколько дорого они нам обходятся?

3. Каналы сбыта:

- через какие каналы наши клиенты хотят получать наше ценностное предложение?

- как они получают его сейчас?

- какие каналы наиболее эффективны?

- насколько они согласуются с предпочтениями потребителей?

- какие из них наименее затратны?

Слева в матрице ВМС расположен блок, описывающий бизнес-структуру компании, ее внутренние ресурсы и ключевых партеров.

4. Ключевые ресурсы:

- какие ключевые ресурсы необходимы для создания ценностного предложения?

- каковы каналы распространения?

- как строятся отношения с клиентами?

- как формируются потоки доходов?

- для кого мы работаем?

- кто для нас самый важный клиент?

5. Ключевые виды деятельности:

- какие ключевые виды деятельности необходимы для создания ценностного предложения?

- что мы делаем для поддержания каналов продвижения?

- как мы действуем, чтобы выстраивать отношения с клиентами?

- что предпринимаем для получения и учета потоков доходов?

6. Ключевые партнеры:

- кто наши ключевые партнеры?

- кто наши ключевые поставщики?

- какие ключевые ресурсы мы от них получаем?

- какие ключевые действия они для нас выполняют?
В нижней части таблицы ВМС два подраздела описывают финансовые потоки: доходы и структуру расходов.

7. Структура издержек:

- каковы наиболее важные затраты, предусмотренные бизнес-моделью?

- какие из ключевых ресурсов являются самыми дорогими?

- какие основные действия относятся к самым затратным?

8. Потоки поступления доходов:

- за что наши клиенты готовы платить?

- за что они платят сейчас?

- как они платят? как они хотят платить?

- какова доля каждого из финансовых потоков в общей сумме дохода?

Многие традиционные бизнес-модели содержат аналогичные или похожие элементы - инновационность инструмента ВМС заключается не в его элементах, а скорее в подходе к разработке или изменению бизнес-модели. Он делает процесс простым, быстрым, итерационным и, в отличие от других подходов, наглядным. Вопросы каждого из элементов ВМС сформулированы таким образом, что позволяют быстро находить ключевые факторы и показатели, влияющие на бизнес. Вся бизнес-модель представлена кратко, на одном листе. Более того, модель Остервальдера дает возможность четко сформулировать и донести до всех в организации, каким образом бизнес-модель может обеспечить конкурентное преимущество. Применение ВМС процесс итерационный: недостаточно создать одну, хоть и законченную модель, важно регулярно переосмысливать текущее положение дел, искать альтернативы и инновационные подходы. С помощью ВМС удобно вести дискуссию по существующей или желаемой бизнес-модели; инструмент позволяет изменять бизнес-модель, тем самым делая ее более адаптивной к потребностям современного мира с его быстро изменяющимися технологиями и предпочтениями потребителей. Иными словами, применение этого инструмента помогает постоянно подвергать сомнению status quo компании.

Поскольку ВМС очень часто используется для разработки бизнес-моделей стартапами, возникло мнение, что этот инструмент больше подходит для поиска новых бизнес- 
моделей, чем для изменения старых. Это не совсем так. Для традиционных компаний инструмент ВМС обычно используется не для определения бизнес-логики всей компании, а для создания нового бизнеса для конкретного продукта или услуги. Когда возникает идея запуска продукта или услуги, ВМС позволяет понять его/ее зависимость от существующей бизнес-модели, факторы успеха и ограничения для успешной реализации идеи. Проводя анализ с помощью ВМС, можно предусмотреть необходимые шаги по продвижению этого продукта или услуги потребителям, правильно его позиционировать и выстроить структуру финансовой поддержки.

ВМС также подходит и для сравнения различных бизнес-моделей между собой. Можно, например, разработать бизнес-модель для продвижения нового продукта или услуги и сопоставить ее с бизнес-моделью конкурента. В рамках такого анализа важно выявить ключевые различия между сравниваемыми моделями. Один и тот же продукт или услуга в разных компаниях может быть предложен потребителю по-разному, опираться на разные бизнес-модели, и успех будет зависеть от того, насколько инновационной и цельной окажется бизнес-модель.

Наиболее эффективно использовать ВMC как часть системного подхода к управлению инновациями. В таком ракурсе ВМС становится одним из шагов процесса быст- рого, адаптивного дизайнирования (agile design process) (рис. 2).

Процесс управления инновациями, нацеленными на результат, имеет 8 этапов. Разрабатывая новые продукты или услуги, важно понять уже на первом этапе, насколько они могут удовлетворить будущие потребности клиентов. На втором этапе необходимо ответить на ключевые вопросы: есть ли у нас ресурсы для разработки этого продукта, этой услуги или необходимо их приобрести, создать, развить внутри компании? Насколько наша стратегия поддерживает разработку? Установлены ли необходимые KPI для достижения стратегических целей и отслеживания процессов? На третьем этапе применяются различные техники для выработки идей по возможным продуктам, услугам, как правило, здесь применяются техники дизайн-мышления. Четвертый этап - «управление портфелем» - помогает получить на выходе оптимальный набор идей и связанных с ними возможных рисков, чтобы определить наиболее многообещающие идеи развития бизнеса и расставить приоритеты. Далее необходимо позаботиться о правах на интеллектуальную собственность, чтобы сохранить конкурентное преимущество. На шестом шаге начинается процесс построения бизнес-модели, где применяется ВМС, а также другие инструменты быстрого прототипирования для переноса идей, концепций в возможные варианты про-

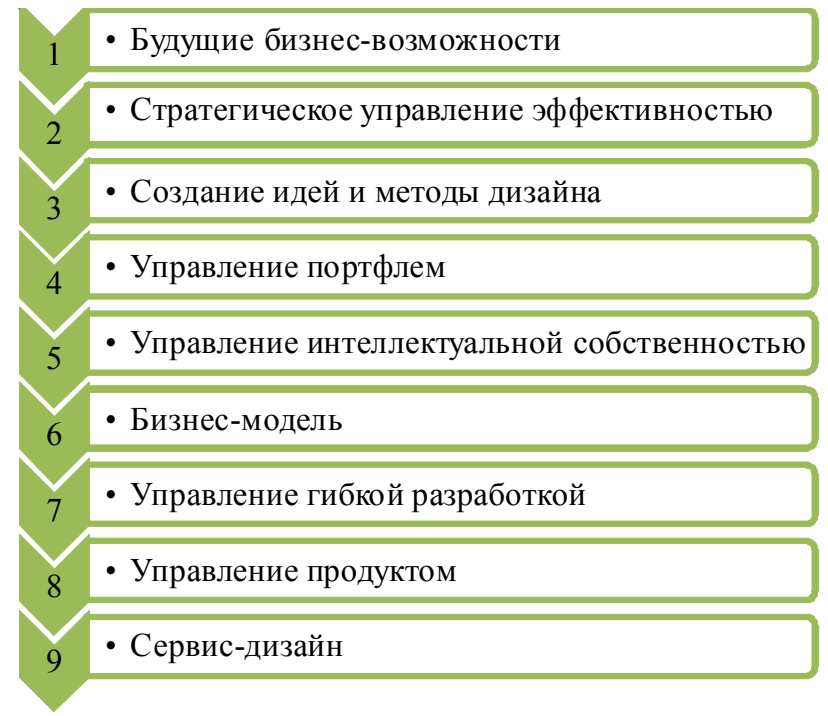

Рис. 2. Бизнес-модель ВМС как часть системного подхода к управлению инновациями

Примечание. Составлено автором на основе источников: [Мариничев, 2015, с. 73; Жанабаев, 2020, с. 151]. 
дуктов или услуг. SRCUM - седьмой этап помогает быстро создать решение, и на завершающем этапе разрабатывается план поддержки продукта или услуги на протяжении всей его/ее жизни, а также необходимые сервисы для формирования уникального клиентского опыта.

Инструмент ВМС достаточно прост в использовании и позволяет быстро определять сущность бизнеса. Тем не менее наивно думать, что этот инструмент не имеет ограничений. Целый мир лежит за пределами бизнеса, постоянно появляются новые тренды, новые конкуренты, которые пока не учтены при анализе. ВМС включает в себя аспекты, которые сложно предсказать. Например, как ответить на вопросы: чего действительно хотят клиенты? возможно ли партнерство с тем или иным агентом на рынке? какова оптимальная ценовая модель? каким образом защитить интеллектуальную собственность и превратить ее в ценностное предложение? Именно поэтому, чтобы более четко сформулировать элементы ВМС, можно использовать другие форматы и модели: анализ трендов и создание форсайтов, методы генерации идей, дизайн-мышление и т. д.

Резюмируя, можно сказать, что ВMC очень важный, но не единственный подход для создания алгоритма, позволяющего добиваться успеха в бизнесе. Больший эффект от применения инструмента возможно получить только комбинируя его с другими инструментами.

\section{Результаты}

Развитие современных технологий позволило гибким IT-компаниям вывести на рынок свои новые продукты и услуги, замещающие традиционные банковские (например, равноправное кредитование, роботизированных советников по управлению личными финансами или полностью цифровые банки без отделений), и тем самым навязать конкуренцию банкам. В то же время стандарты регулирования традиционных банков после кризиса 2008 г. ужесточились, мировой рынок банковского кредитования сузился, а новые директивы угрожают сохранению еще одного конкурентного преимущества бан- ков - монополии на огромные базы данных о клиентах. Меняется и поведение потребителей, которым важно не только удовлетворить свои материальные потребности, но и найти способ самореализации в качестве изобретателя, создателя и производителя новой услуги или продукции. Например, используя Facebook, пользователь одновременно выступает и как потребитель, и как создатель контента, причем самореализация через публикацию собственного контента для него важнее, чем потребление чужого. Платформы завоевывают все большую долю рынка в разных отраслях [KPMG ...; IBM ...]. Однако, чтобы стать успешной платформой, недостаточно только соединять производителей и потребителей ценностей. Alibaba Group, Apple, Uber, AirbnB, Amazon, Facebook, Googleвсе эти успешные IT-компании навсегда трансформировали свои рынки, не просто соединяя производителя с потребителем, но и создавая дополнительную ценность для всех участников платформы.

Рассмотрим опыт китайской компании Alibaba Group, использующей для развития бизнеса ключевую потребность зарубежных клиентов в платформе, на которой были бы не просто представлены китайские производители из совершенно разных отраслей, но также и проведена проверка на реальное существование этих производителей и их соответствие рекламным заявлениям. Проверяя производителей и присваивая им на основании результатов проверки статус «золотого партнера», Alibaba помогает устанавливать доверительные отношения между китайскими производителями и зарубежными клиентами, а также зарабатывает выручку, взимая плату с производителей за осуществление проверки. В отличие от Amazon, y Alibaba нет собственных складов, компания предоставляет своим партнерам только платформу, что позволяет ей быстро масштабироваться на новые рынки. Помимо этого, Alibaba подключает к своей платформе различных провайдеров логистических и складских услуг, тем самым предоставляя своим партнерам больший выбор провайдеров, которые конкурируют между собой, зарабатывая на комиссии за каждый размещенный заказ и рекламу внутри платформы [Pontefract Dan ...]. 
Банковская сфера также не стоит на месте, и в ближайшем будущем новые игроки могут полностью разрушить привычные правила нашей отрасли. Чтобы не просто выжить, но и обеспечить рост собственного капитала в долгосрочной перспективе, банкам необходимо проактивно начать трансформацию бизнес-моделей.

С точки зрения банковского бизнеса можно выделить три ключевые вариации бизнес-модели:

1. «Провайдер инфраструктуры». Данная бизнес-модель построена на использовании облачных технологий для предоставления четырех ключевых услуг: осуществление расчетов, отчетность перед регулятором, процессинг платежей, а также поддержка и обслуживание сервисов и продуктов партнеров через интеграцию по протоколу АРІ. Банк, использующий данную бизнес-модель, должен не просто предоставлять свою инфраструктуру, связанную с облачными технологиями, но и по возможности создавать дополнительную ценность для клиентов (например, скоринговые алгоритмы) или определять и производить ребалансировку уровня риска банковской книги и так далее. Бизнес-модель «Провайдер инфраструктуры» нацелена на максимизацию эффекта экономии от масштаба имеющейся у банка инфраструктуры, а также на высокую степень автоматизации внутренних процессов. Однако, поскольку в такой модели нет места для участия пользователей и привлечения их к цепочке создания добавленной стоимости, она не является высокомаржинальной. Учитывая также национальные особенности банковского регулирования, подобные банки поставщики инфраструктуры, скорее всего, будут иметь национальное значение, не масштабируемое на мировой рынок.

По такой бизнес-модели уже работают некоторые банки, например The Bancorp, который специализируется на предоставлении инфраструктуры для процессинга платежей, выпуске карт различных платежных систем для банков, разработке систем для трастовых фондов, позволяющих осуществлять планирование и анализ инвестиций клиентов, внедрение и интеграцию инновационных решений (например, в области управления активами или осуществления платежей) в существующую инфраструктуру банка. Также данная бизнесмодель и аналогичные сервисы предоставляются конкурентом The Bancorp - solarisBank.

Рассмотрим результат применения бизнес-модели «Провайдер инфраструктуры» в других индустриях. Так, датская компания LEGO, выпускающая конструкторы для детей, применила ее в В2С сегменте, предоставляя конечным клиентам инфраструктуру (включая пользовательский интерфейс) для создания конструкторов с собственным дизайном с использованием блоков, которые производит компания, что вовлекает пользователей в процесс создания конечного продукта. Дети чувствуют, что, собирая конструктор по собственному дизайну, они создают нечто уникальное, чем можно будет гордиться перед сверстниками, что создает дополнительную ценность.

2. «Агрегатор». Данная бизнес-модель предполагает предоставление потребителям максимально широкого и персонализированного, благодаря использованию алгоритмов искусственного интеллекта, выбора.

Для того чтобы реализовать эту бизнесмодель, банк должен стать для потребителей виртуальным помощником, который на основе анализа данных о клиенте соединяет его с наиболее подходящим провайдером под конкретную потребность. Как, например, агрегатор авиабилетов компания Aviasales на основании данных о желаемом маршруте перелета (с пересадкой или без), классе салона, предпочитаемой авиакомпании, аэропорте пересадки, а также других фильтров подбирает наиболее выгодные авиабилеты, которые подходят под потребности клиента [NetDragon ...]. Таким же образом и банк-агрегатор должен находить наиболее подходящего провайдера финансовой услуги, предоставляя дополнительную ценность для конечного потребителя, анализируя соответствие предложений различных провайдеров и профиль клиента на основе данных о его транзакциях. Выручка при использовании данной бизнес-модели формируется за счет поступлений от сравнительно невысокого комиссионного вознаграждения за операции, осуществляемые клиентами. Данная комиссия взимается либо с самих клиентов, либо с партнеров агрегатора, размещающих свои услуги на их площадке. 
Основная проблема использования данной бизнес-модели в банковском секторе заключается в закрытости данных о транзакциях клиентов внутри банков. Без доступа к информации практически невозможно создать алгоритм машинного обучения, который бы делал правильные выводы и, как следствие, давал подходящие советы. В этих условиях значительную роль должна сыграть европейская директива PSD2, открывающая третьим лицам доступ к транзакционным данным клиентов (с их согласия). Получив доступ к данным о потребительском поведении пользователей, агрегаторы будут предоставлять ценность не только благодаря сравнению стоимости различных провайдеров банковских услуг и отзывов клиентов (как, например, сегодня делает портал Banki.ru), но и благодаря ценным советам о том, каким сервисом лучше воспользоваться конкретному человеку в конкретной ситуации, анализируя множество факторов помимо цены. В дополнение к умным советам на основе анализа данных эта бизнес-модель предполагает развитые цифровые каналы распространения ценности, геймификацию процессов взаимодействия с пользователем, мощную CRM-систему, открытый каталог продуктов и сервисов и открытый API для интеграции с партнерами.

Рассмотрим применение бизнес-модели «Агрегатор» в других индустриях. Так, агрегатор отелей Booking.com произвел революцию на рынке гостеприимства. Если раньше большинство бронирований осуществлялось через агентства или напрямую в отеле, то Booking.com позволил клиентам сравнивать огромное количество предложений различных отелей, отбирая наиболее подходящие с помощью фильтров, а также анализируя отзывы миллионов других путешественников с различными демографическими характеристиками и целями поездки, чтобы клиент мог просматривать наиболее релевантные отели и отзывы.

3. «Открытая платформа». Данная бизнес-модель вертикально интегрированного банка с открытой платформой предполагает предложение узкого набора собственных стратегически важных услуг, традиционно вызывающих доверие и спрос у клиентов, например, ведение текущих счетов и открытие де- позитов [доступ к относительно дешевому фондированию, осуществление платежей, обеспеченные кредиты (как вид кредитования, в котором традиционные банки обладают существенными конкурентными преимуществами, достигаемыми за счет экспертизы и экономии от масштаба)] и т. д. В то же время данная бизнес-модель предполагает одновременное использование возможностей финансового супермаркета как в модели банковского агрегатора, предлагая услуги сторонних провайдеров. Ключевые продукты, которые могут быть предложены сторонними провайдерами в рамках данной бизнесмодели, следующие: электронные кошельки, необеспеченное равноправное кредитование физических лиц, аналитика личных и корпоративных финансов, кредитование малого и среднего бизнеса на основе аналитики денежных потоков, трансграничные переводы, роботизированные инвестиции и социальный трейдинг.

Рассмотрим результат применения бизнес-модели «Открытая платформа» в других индустриях. Так, компания Uber, чистая выручка которой в 2016 г. достигла 6,5 млрд долл., полностью трансформировала рынок частных перевозок. Сократив во много раз время подачи автомобиля и стоимость поездки за счет подключения к своей платформе огромного количества водителей, которые хотят зарабатывать в свободное время, компания Uber добилась лояльности клиентов и вытеснила конкурентов (традиционные таксопарки) с рынка. В то же время у Uber нет своих автомобилей и нет водителей в штате, это полностью диджитализированная компания, благодаря чему ее масштабирование на другие рынки происходит очень быстрыми темпами. Сегодня Uber работает уже в 570 городах по всему миру [Technavio ...].

Ключевым отличием бизнес-модели «открытая платформа» в банковской сфере от других рынков, например, частных перевозок, на котором работает Uber, заключается в необходимости предоставления собственных услуг, а не только соединения и оркестрирования взаимодействий между поставщиками и потребителями ценности, что предполагает необходимость получения банковской лицензии, развитие компетенций риск-менеджмента, комплаенса, предоставление банковской 
отчетности регулятору и т. д. В то же время необходимо учитывать и готовиться к тому, что переход на платформенную бизнес-модель предполагает тотальную трансформацию работы всех подразделений, существенный upgrade информационных систем как с точки зрения безопасности, так и с точки зрения сбора и аналитики данных. Несмотря на сложности, присущие данным процессам, трансформация бизнес-модели и адаптация к новой, изменившейся среде - необходимые условия для роста в долгосрочной перспективе.

\section{Выводы}

Формирование бизнес-модели - процесс итерационный: недостаточно создать одну, хоть и законченную модель, важно регулярно переосмысливать текущее положение дел, искать альтернативы и инновационные подходы.

Дискуссии по выбору существующей или желаемой бизнес-модели будут продолжаться, поэтому описанные инструменты позволят изменять бизнес-модель, тем самым делая ее более адаптивной к потребностям современного мира с его быстро изменяющимися технологиями и предпочтениями потребителей.

Практическая значимость исследования заключается в том, что полученные результаты позволят компаниям добиваться успеха в бизнесе, получая больший эффект от применения данной бизнес-модели, комбинируя еe с другими инструментами.

Это поможет компаниям получить на выходе оптимальный набор идей и связанных с ними возможных рисков, чтобы определить наиболее многообещающие идеи развития бизнеса и расставить приоритеты. Несмотря на сложности, присущие данным процессам, трансформация бизнес-модели и адаптация к новой, изменившейся среде - необходимые условия для роста в долгосрочной перспективе.

\section{СПИСОК ЛИТЕРАТУРЫ}

Абанина, И. Н. Трансформация бизнес-моделей в цифровой экономике / И. Н. Абанина // Caмоуправление. -2019. - Т. 2, №2 (115). - С. 2-4.

Благов, Е. Ю. Платформенные бизнес-модели компаний экосистемы национальной технологи- ческой инициативы / Е. Ю. Благов, Н. И. Кулаева // Вопросы инновационной экономики. 2020. - Т. 10, № 1. - С. 157-172.

Дунаев, О. Управление изменениями бизнес-моделей компаний / О. Дунаев // Логистика. - 2020. № 8(165). - C. 29-31.

Жанабаев, Е. Е. Лы. Моделирование и управление бизнес-процессами / Е. Е. Лы. Жанабаев // Актуальные научные исследования в современном мире. - 2020. - № 10-1 (66). - С. 151-155.

Мариничев, В. А. Опыт зарубежных компаний по разработке и реализации стратегически ориентированных бизнес-моделей / В. А. Мариничев // Экономические науки. - 2015. № 129. - C. 73-77.

IBM Institute for Business Value. Facing the storm. Navigating the global skills crisis. 2016. - Электрон. текстовые дан. - Режим доступа: http:// blog.oxfordeconomics.com/facing-the-stormnavigating-the-global-skills-crisis (дата обращения: 19.08.2020). - Загл. с экрана.

KPMG. Corporate Digital Learning. - Электрон. текстовые дан. - Режим доступа: https:// iversity.org/en/courses/corporate-digitallearning (дата обращения: 19.08.2020). - Загл. с экрана.

Pontefract Dan. Pervasive Learning Graphic from Flat. - Электрон. текстовые дан. - Режим доступа: https://www.danpontefract.com/ pervasive-learning-graphic-from-flat-army (дата обращения: 02.10.2018). - Загл. с экрана.

NetDragon. Announces 2019 Interim Financial Results, Record First Half Revenue, Profit more than Doubled. - Электрон. текстовые дан. - Режим доступа: http://www.netdragon.com (дата обращения: 19.08.2020). - Загл. с экрана.

Technavio. Online Language Learning Market in the US 2018-2022. Increased Use of VR to Boost Demand. - Электрон. текстовые дан. - Режим доступа: https://www.technavio.com (дата обращения: 19.08.2020). - Загл. с экрана.

\section{REFERENCES}

Abanina I.N. Transformaciya biznes-modelej v cifrovoj ekonomike [Transformation of Business Models in the Digital Economy]. Samoupravlenie [SelfGovernment], 2019, vol. 2, no. 2 (115), pp. 2-4.

Blagov E.Yu., Kulaeva N.I. Platformennye biznesmodeli kompanij ekosistemy nacional'noj tekhnologicheskoj iniciativy [Platform Business Models of Companies in the Ecosystem of the National Technological Initiative]. Voprosy innovacionnoj ekonomiki [Issues of Innovative Economics], 2020, vol. 10, no. 1, pp. 157-172. 


\section{УПРАВЛЕНИЕ ЭКОНОМИЧЕСКИМ РАЗВИТИЕМ}

Dunaev O. Upravlenie izmeneniyami biznes-modelej kompanij [Management of Changes in Business Models of Companies]. Logistika [Logistics], 2020, no. 8 (165), pp. 29-31.

Zhanabaev E.E. Ly. Modelirovanie i upravlenie biznesprocessami [Modeling and Management of Business Processes]. Aktual'nye nauchnye issledovaniya $v$ sovremennom mire [Actual Scientific Research in the Modern World], 2020, no. 10-1 (66), pp. 151-155.

Marinichev V.A. Opyt zarubezhnyh kompanij porazrabotke i realizacii strategicheski orientirovannyh biznesmodelej [Experience of Foreign Companies in the Development and Implementation of Strategically Oriented Business Models]. Ekonomicheskie nauki [Economic Sciences], 2015, no. 129, pp. 73-77.

IBM Institute for Business Value. Facing the Storm. Navigating the Global Skills Crisis. 2016. URL: http://blog.oxfordeconomics.com/facing-thestorm-navigating-the-global-skills-crisis (accessed 19 August 2020).

KPMG. Corporate Digital Learning. URL: https:// iversity.org/en/courses/corporate-digitallearning (accessed 19 August 2020).

Pontefract Dan. Pervasive Learning Graphic from Flat. URL: https://www.danpontefract.com/ pervasive-learning-graphic-from-flat-army (accessed 2 October 2018).

NetDragon. Announces 2019 Interim Financial Results, Record First Half Revenue, Profit More Than Doubled. URL: http://www.netdragon. com/ (accessed 19 August 2020).

Technavio. Online Language Learning Market in the US 2018-2022. Increased Use of VR to Boost Demand. URL: https:/www.technavio.com/ (accessed 19 August 2020).

\section{Information About the Author}

Elena V. Shirinkina, Candidate of Sciences (Economics), Head of the Department of Management and Business, Surgut State University, Lenina St, 1,628412 Surgut, Russian Federation, shirinkina86@yandex.ru, https://orcid.org/0000-0002-6933-1903

\section{Информация об авторе}

Елена Викторовна Ширинкина, кандидат экономических наук, заведующий кафедрой менеджмента и бизнеса, Сургутский государственный университет, ул. Ленина, 1, 628412 г. Сургут, Российская Федерация, shirinkina86@yandex.ru, https://orcid.org/0000-0002-6933-1903 